\title{
Previsão de Curvas de Titulação: uma abordagem quantitativa
}

\author{
J. L. G. F. S. Costa Pereira*
}

Nester

este trabalho procurou-se dar uma perspectiva matemático-numérica a um problema de Química com diversas aplicações. Para esse efeito, foram deduzidos e propostos os modelos de quantificação em cada caso de titulação ácido-base com espécies monopróticas. Os modelos aqui desenvolvidos permitem a sua fácil utilização quer ao nível da previsão de curvas de titulação potenciométrica quer da estimativa de propriedades relacionadas com o comportamento ácido-base. Este trabalho procura ser um contributo para questões analíticas e educacionais relacionadas com o equilíbrio ácido-base em meio aquoso sendo aqui analisados quatro casos genéricos, relacionados com a titulação de espécies monopróticas em meio aquoso: a) titulação de ácido forte com base forte, b) titulação de ácido fraco com base forte, c) titulação de ácido forte com base fraca e d) titulação de ácido fraco com base fraca. Alguns destes exemplos estão acompanhados de demonstração através de uma folha de cálculo.

Palavras-chave: curvas titulação potenciométrica, modelos, previsão, reacções ácido-base

\section{INTRODUÇ̃̃O}

Devido às propriedades ácido-base de um grande número de compostos, as curvas de titulação ácido-base, seguidas por via potenciométrica ${ }^{1}$, constituem um procedimento laboratorial relativamente simples e muito importante, quer no campo analítico (na determinação de analitos), bem como no campo termodinâmico (possibilitando a estimativa de constantes de equilíbrio). Estas constantes por sua vez são imprescindíveis para inúmeras aplicações, desde as ambientais (relacionadas com a especiação de elementos), passando pelas industriais (estabilização de polímeros e misturas coloidais como tintas, detergentes, etc), farmacêuticas (estabilização de formulações, libertação controlada de fármacos), biomédicas (polímeros biocompatíveis, bio-géis, transdérmicos, etc).

A previsão de curvas de titulação é deveras importante, quer para permitir a escolha do melhor indicador para a titulação, quer para verificar, confirmar ou estimar resultados com base nos resultados experimentais.

\footnotetext{
* Departamento de Química da Faculdade de Ciências e Tecnologia da Universidade de Coimbra 3004-535 Coimbra, Portugal e-mail: jcpereira@qui.uc.pt
}

O objectivo deste trabalho é o de mostrar como se pode prever uma curva de titulação com base numa perspectiva quantificadora. Entendemos que este assunto em termos numéricos já é por si só algo difícil, razão pela qual, para não dificultar a sua leitura, não iremos entrar em minucioso detalhe teórico quanto a definições e fundamentação do equilíbrio ácido-base, daí as subsequentes simplificações nesta abordagem.

Sendo este tipo de reacções muito genérico e simples não é por acaso que os alunos de Química começam por aprender o equilíbrio ácido-base. Ainda assim, este conceito fica por vezes mal assimilado pelos alunos uma vez que existem diferentes abordagens possíveis para o tratamento do equilíbrio ácido-base [1, 2].

Neste trabalho vamos adoptar a teoria ácido-base proposta em 1923 por Johannes Nicolaus Brønsted e Thomas Martin Lowry (teoria de Brønsted-Lowry) já que esta é mais simples e válida para entender a transferência de protão entre espécies em meio aquoso.

Segundo a teoria proposta por Brønsted-Lowry as reacções de ácido-base podem ser descritas em poucos postulados sendo os seguintes os mais fundamentais:
1. Um ácido é uma espécie química que doa um protão $\left(\mathrm{H}^{+}\right)$;

2. Uma base é uma espécie química que recebe um protão;

3. Uma espécie ácida possui uma base conjugada associada;

4. Uma espécie básica possui um ácido conjugado associado;

5. Um ácido $(H A)$ reage com uma base (B) por transferência de protão originando as respectivas espécies conjugadas $\left(\mathrm{A}^{-}\right.$e $\left.\mathrm{BH}^{+}\right)$.

Os modelos que são aqui descritos têm em conta o procedimento experimental mais adequado com vista a que estes possam servir para descrever os resultados experimentais.

Assim sendo, antes de mais, convém referir que, para minimizar os erros de carbonatação ${ }^{2}$ das soluções do titulante $^{3}$ e do titulado ${ }^{4}$, o processo deve ser conduzido experimentalmente utilizando o ácido como titulado e a base como titulante - deste modo o contacto do ar com a base é minimizado e a agitação da solução de titulado (para facilitar o equilíbrio químico) não sofre muita contaminação se a titulação for realizada rapidamente ${ }^{5}$. A titulação potenciométrica é conduzida com um sistema potenciométrico sensível ao hidrogenião imerso na solução de titulado com um sistema de agitação adequado ${ }^{6}$. 
Ao nível do ensino opta-se por escoIher uma perspectiva simplista da titulação que permite obter um traçado algo indefinido como aproximação da curva de titulação.

A descrição do procedimento para estimativa da curva de titulação envolvendo a) ácido forte $(25,00 \mathrm{~mL} \mathrm{HCl}$ $0,100 \mathrm{M})$ com uma base forte $(\mathrm{NaOH}$ $0,100 \mathrm{M}), \mathrm{b})$ ácido fraco $(25,00 \mathrm{~mL}$ $\mathrm{CH}_{3} \mathrm{COOH} 0,100 \mathrm{M}$ ) com uma base forte $(\mathrm{NaOH} \mathrm{0,100} \mathrm{M)} \mathrm{e} \mathrm{c)} \mathrm{ácido} \mathrm{for-}$ te $(25,00 \mathrm{~mL} \mathrm{HCl} \mathrm{0,100} \mathrm{M)} \mathrm{com} \mathrm{uma}$ base fraca $\left(\mathrm{NH}_{3} 0,100 \mathrm{M}\right)$ encontra-se descrito em livros de base para Química Geral [1].

De acordo com esta perspectiva, no caso das espécies monopróticas ${ }^{7}$ anteriormente especificadas, este cálculo, por razões que serão posteriormente apresentadas, está fragmentado em quatro etapas sequenciais:

a) antes de qualquer adição $\left(\mathrm{V}_{\mathrm{b}}=0,00\right.$ $\mathrm{mL}$ ) - o pH da solução do titulado é condicionado pela ionização do ácido;

b) na zona tamponizante antes do volume de equivalência - $\mathrm{pH}$ determinado pelo par ácido-base do titulado;

c) no ponto de equivalência - onde existe proporcionalidade estequiométrica entre o titulante e o titulado;

d) após o ponto de equivalência - $\mathrm{pH}$ determinado pelo par ácido-base do titulante.

Esta segmentação do problema é vantajosa porque permite obter, de uma forma simples, um traçado algo grosseiro da curva de titulação - a zona tamponizante ${ }^{8}$ encontra-se restringida entre $1 / 10$ e $9 / 10$ do valor do volume de equivalência ${ }^{9}$ e passa a ser desconhecida sobretudo próximo do ponto de viragem ${ }^{10}$ onde as aproximações efectuadas deixam de ser válidas. Contudo, esta abordagem é perigosa em termos educativos já que induz o aluno a assumir uma descontinuidade no sistema físico-químico em estudo.

A abordagem completa deste tipo de situações já foi há muito tempo descrita, perdendo-se na história a sua origem, mas pode ser encontrada compilada de uma forma sistemática [3].
Esta abordagem baseia-se essencialmente em dois princípios aplicáveis às soluções de electrólito em sistema fechado:

a) balanço de massa - as espécies formadas em solução dependem estequiometricamente da quantidade total dissolvida;

b) balanço de carga - as soluções de electrólito são electricamente neutras devido a uma compensação estequiométrica das cargas dos iões presentes em solução.

As equações resultantes são, regra geral, difíceis de resolver, pois frequentemente atingem grau superior a três. A proposta deste artigo é facultar ao seu leitor um modo de resolver esse problema de modo numérico iterativo, servindo-se de uma interface gráfica como auxiliar, o programa Microsoft Excel.

Na primeira parte deste trabalho serão deduzidas as equações que descrevem a curva de titulação e na segunda parte serão realizadas demonstrações e testes com estes modelos.

\section{Aproximações e Fundamentação}

No sentido de minimizar os erros de carbonatação assume-se que o titulado é sempre um ácido e o titulante uma base. Assume-se ainda que, durante a titulação, as constantes de equilíbrio (produto iónico da água e constantes de ionização) não variam ${ }^{11}$.

Os valores das constantes a utilizar podem reportar-se aos valores termodinâmicos (válidas para soluções muito diluídas) ou constantes condicionais adequadas à experiência em causa - a curva de titulação prevista terá maior fiabilidade quanto mais correctos estiverem estes valores.

Assume-se também que as reacções em meio aquoso são rápidas, permitindo que, em qualquer instante da curva de titulação, a concentração das diversas espécies presentes na solução do titulado possa ser descrita através das respectivas constantes de equilíbrio.

Em termos de notação, um ácido forte é representado por $\mathrm{HX}$ e um ácido fraco por $\mathrm{HA}$ sendo $\mathrm{C}_{\mathrm{a}}$ e $\mathrm{V}_{\mathrm{a}}$ a respectiva concentração e volume pipetado. No caso de uma base forte esta representa-se por $\mathrm{MOH}$ enquanto que uma base fraca por $B$ sendo $C_{b}$ e $V_{b}$ a respectiva concentração e volume vertido.

No caso de reagentes monopróticos, no ponto de equivalência $\left(V_{b}=V_{e q}\right)$ atinge-se a igualdade estequiométrica entre as moles de ácido presentes no titulado e da base adicionada através da bureta,

$C_{a} \cdot V_{a}=C_{b} \cdot V_{\text {eq }}$

Quanto aos equilíbrios em meio aquoso serão considerados apenas o produto iónico da água $\left(\mathrm{K}_{\mathrm{w}}\right)$ e as constantes de acidez $\left(\mathrm{K}_{\mathrm{a}}\right)$. Para facilitar o tratamento matemático, estas serão aqui consideradas como produtos e/ou quocientes de concentrações analíticas.

Com base nesta aproximação, o produto iónico da água é dado por

$$
\mathrm{K}_{\mathrm{w}}=\left[\mathrm{H}^{+}\right]\left[\mathrm{OH}^{-}\right]
$$

e a constante de acidez de um ácido fraco (HA) é dada pelo quociente

$\mathrm{K}_{\mathrm{a}}=\frac{\left[\mathrm{H}^{+}\right]\left[\mathrm{A}^{-}\right]}{[\mathrm{HA}]}$

No sentido de uniformizar este tratamento matemático, as constantes de basicidade $\left(\mathrm{K}_{\mathrm{b}}\right)$ devem ser convertidas na respectiva constante de acidez através de

$\mathrm{K}_{\mathrm{a}}=\frac{\mathrm{K}_{\mathrm{w}}}{\mathrm{K}_{\mathrm{b}}}$

Em qualquer instante da titulação o valor de $\mathrm{pH}$ da solução pode ser estimado com base na concentração analítica do hidrogenião $\left(\mathrm{H}^{+}\right)$

$$
-\log \left(a_{\mathrm{H}^{+}}\right)=\mathrm{pH} \approx-\log \left(\left[\mathrm{H}^{+}\right]\right)
$$

Assume-se que em solução diluída ( $C$ « $1 \mathrm{M})$ os electrólitos fortes $(\mathrm{HX}, \mathrm{MOH}$ e MX) encontram-se totalmente ionizados, sendo os iões $\mathrm{M}^{+}$e $\mathrm{X}^{-}$inertes em termos de comportamento ácido-base, permanecendo em solução sob a forma hidratada. Considera-se também que o coeficiente de activida- 
de das espécies envolvidas é igual à unidade.

No caso de um ácido fraco monoprótico $(\mathrm{HA})$, o balanço de massa é dado por

$$
C_{a}=[\mathrm{HA}]+\left[\mathrm{A}^{-}\right]
$$

e as respectivas fracções molares da espécies são

$$
\alpha_{0}=\frac{[\mathrm{HA}]}{\mathrm{C}_{\mathrm{a}}}=\left(\frac{\left[\mathrm{H}^{+}\right]}{\left[\mathrm{H}^{+}\right]+\mathrm{K}_{\mathrm{a}}}\right)
$$

e

$$
\alpha_{1}=\frac{\left[\mathrm{A}^{-}\right]}{\mathrm{C}_{\mathrm{a}}}=\left(\frac{\mathrm{K}_{\mathrm{a}}}{\left[\mathrm{H}^{+}\right]+\mathrm{K}_{\mathrm{a}}}\right)
$$

De modo similar, o balanço mássico de uma base fraca (B) em meio aquoso é dada por

$$
\mathrm{C}_{\mathrm{b}}=\left[\mathrm{HB}^{+}\right]+[\mathrm{B}]
$$

e as respectivas fracções molares da espécies são

$$
\alpha_{0}=\frac{\left[\mathrm{HB}^{+}\right]}{\mathrm{C}_{\mathrm{b}}}=\left(\frac{\left[\mathrm{H}^{+}\right]}{\left[\mathrm{H}^{+}\right]+\mathrm{K}_{\mathrm{a}}}\right)
$$

e

$$
\alpha_{1}=\frac{[B]}{C_{b}}=\left(\frac{\mathrm{K}_{a}}{\left[\mathrm{H}^{+}\right]+\mathrm{K}_{\mathrm{a}}}\right)
$$

Do ponto de vista da solução do titulado, esta corresponde a um sistema aberto já que diversos incrementos de volume de titulante são progressivamente adicionados, indo aumentar o volume da solução final. Este facto exige que todas a concentrações sejam actualizadas em cada instante pelo respectivo factor de diluição - no instante $i$ da titulação, a concentração total de ácido no titulado $\left(C_{a}(i)\right)$ é dada por

$$
C_{a}(i)=C_{a} \cdot\left(\frac{V_{a}}{V_{a}+V_{b}}\right)
$$

Enquanto que a concentração total de base adicionada nesse instante $\left(\mathrm{C}_{\mathrm{b}}(\mathrm{i})\right)$ é dada por

$$
C_{b}(i)=C_{b} \cdot\left(\frac{V_{b}}{V_{a}+V_{b}}\right)
$$

Estas aproximações e pressupostos permitem deduzir os seguintes mode- los para a previsão e modelação de curvas de titulação.

No caso da previsão de curvas de titulação assumem-se como determinados os valores das concentrações das espécies $\left(C_{a}, C_{b}\right)$ e os respectivos volumes $\left(V_{a}, V_{b}\right)$ bem como as constantes de equilíbrio sendo estimado o valor do $\mathrm{pH}$ em cada instante da titulação.

No caso da modelação, regra geral, conhecem-se os volumes $\left(\mathrm{V}_{\mathrm{a}}, \mathrm{V}_{\mathrm{b}}\right)$ e pelo menos uma concentração $\left(C_{a}\right.$ ou $\mathrm{C}_{\mathrm{b}}$ ) e o valor do $\mathrm{pH}$ em cada instante da curva de titulação. Neste caso procura-se estimar as constantes de equilíbrio e a outra concentração (ou volume equivalente, $\mathrm{V}_{\text {eq }}$ ).

Neste trabalho são analisados quatro casos genéricos relacionados com a utilização exclusiva de espécies monopróticas: a) titulação de ácido forte com base forte, b) titulação de ácido fraco com base forte, c) titulação de ácido forte com base fraca e d) titulação de ácido fraco com base fraca.

\section{Caso 1 - Titulação de Ácido forte (HX) COM UMA BASE FORTE (MOH)}

Nesta situação a reacção química em meio aquoso pode ser indicada sob a forma

$$
\mathrm{H}^{+}(\mathrm{aq})+\mathrm{OH}^{-}(\mathrm{aq}) \rightarrow \mathrm{H}_{2} \mathrm{O}(\mathrm{I})
$$

já que os respectivos contra-iões dos electrólitos fortes devem permanecer sob a forma hidratada em solução.

Inicialmente existe um volume $\mathrm{V}_{\mathrm{a}}$ de ácido com concentração $C_{a}$ no vaso de titulação ${ }^{12}$. Após a adição do volume $V_{b}$ de titulante com concentração $\mathrm{C}_{\mathrm{b}}$ o equilíbrio iónico estabelecido na solução titulada é dado por:

$$
\left[\mathrm{H}^{+}\right]+\left[\mathrm{M}^{+}\right]=\left[\mathrm{OH}^{-}\right]+\left[\mathrm{X}^{-}\right]
$$

sendo as concentrações molares dadas por

$$
\begin{aligned}
& {\left[\mathrm{M}^{+}\right]=\left(\frac{\mathrm{C}_{b} \cdot \mathrm{V}_{\mathrm{b}}}{\mathrm{V}_{\mathrm{a}}+\mathrm{V}_{\mathrm{b}}}\right)} \\
& {\left[\mathrm{X}^{-}\right]=\left(\frac{\mathrm{C}_{\mathrm{a}} \cdot \mathrm{V}_{\mathrm{a}}}{\mathrm{V}_{\mathrm{a}}+\mathrm{V}_{\mathrm{b}}}\right)}
\end{aligned}
$$

Por substituição de (0.2) e (1.2) em (1.1) fica-se com,

$$
\left[\mathrm{H}^{+}\right]+\left(\frac{\mathrm{C}_{\mathrm{b}} \cdot \mathrm{V}_{\mathrm{b}}}{\mathrm{V}_{\mathrm{a}}+\mathrm{V}_{\mathrm{b}}}\right)=\frac{\mathrm{K}_{\mathrm{w}}}{\left[\mathrm{H}^{+}\right]}+\left(\frac{\mathrm{C}_{\mathrm{a}} \cdot \mathrm{V}_{\mathrm{a}}}{\mathrm{V}_{\mathrm{a}}+\mathrm{V}_{\mathrm{b}}}\right)
$$

que, pode ser simplificada para dar

$$
\left(\left[\mathrm{H}^{+}\right]-\frac{\mathrm{K}_{\mathrm{w}}}{\left[\mathrm{H}^{+}\right]}\right)+\left(\frac{\mathrm{C}_{\mathrm{b}} \cdot\left(\mathrm{V}_{\mathrm{b}}-\mathrm{V}_{\text {eq }}\right)}{\mathrm{V}_{\mathrm{a}}+\mathrm{V}_{\mathrm{b}}}\right)=0
$$

No caso da previsão da curva de titulação, a equação (1.4) corresponde a uma equação de segundo grau em relação à concentração hidrogeniónica $\left(\mathrm{H}^{+}\right)$

$$
\left[\mathrm{H}^{+}\right]^{2}+\left(\frac{\mathrm{C}_{\mathrm{b}} \cdot\left(\mathrm{V}_{\mathrm{b}}-\mathrm{V}_{\text {eq }}\right)}{\mathrm{V}_{\mathrm{a}}+\mathrm{V}_{\mathrm{b}}}\right) \cdot\left[\mathrm{H}^{+}\right]-\mathrm{K}_{\mathrm{w}}=0
$$

A cada incremento de volume de titulante adicionado $\left(V_{b}\right)$ esta equação pode ser resolvida segundo a fórmula resolvente

$$
\left[\mathrm{H}^{+}\right]=\frac{-\phi+\sqrt{\phi^{2}+4 \mathrm{~K}_{\mathrm{w}}}}{2}
$$

sendo $\phi$ a fracção de concentração titulada

$$
\phi=\frac{\mathrm{C}_{\mathrm{b}} \cdot\left(\mathrm{V}_{\mathrm{b}}-\mathrm{V}_{\mathrm{eq}}\right)}{\mathrm{V}_{\mathrm{a}}+\mathrm{V}_{\mathrm{b}}}
$$

Neste simples exemplo a curva de titulação é resolvida exactamente sem necessidade de qualquer aproximação ou algoritmo iterativo. O procedimento de estimativa corresponde a:

a) estabelecer-se uma escala adequa$\mathrm{da}^{13}$ de volumes que ultrapassem $\mathrm{o}$ volume equivalente,

b) calcular-se a fracção titulada, $\phi$, eq.(1.7),

c) calcular-se a concentração do ião $\mathrm{H}^{+}$, eq.(1.6),

d) estimar-se o valor de $\mathrm{pH}$ respectivo, eq.(0.5),

e) traçar-se a curva de titulação $\mathrm{pH}$ em função do volume de titulante, $\mathrm{V}_{\mathrm{b}}$.

Caso 2 - Titulação de ÁCido Fraco (HA) COM UMA BASE FORTE (MOH)

Nesta situação a reacção química em causa pode ser indicada sob a seguinte forma: 


$$
\begin{aligned}
& \mathrm{HA}^{(\mathrm{aq})}+\mathrm{OH}^{-}(\mathrm{aq}) \rightarrow \\
& \rightarrow \mathrm{A}^{-}(\mathrm{aq})+\mathrm{H}_{2} \mathrm{O}(\mathrm{I})
\end{aligned}
$$

Em qualquer instante da curva de titulação o equilíbrio iónico em causa é dado por

$$
\left[\mathrm{H}^{+}\right]+\left[\mathrm{M}^{+}\right]=\left[\mathrm{OH}^{-}\right]+\left[\mathrm{A}^{-}\right]
$$

Por sua vez, recorrendo às expressões de fracção molar da espécie ácida ionizada, eq.(0.7), a equação anterior converte-se em

$$
\left[\mathrm{H}^{+}\right]+\left(\frac{\mathrm{C}_{\mathrm{b}} \cdot \mathrm{V}_{\mathrm{b}}}{\mathrm{V}_{\mathrm{a}}+\mathrm{V}_{\mathrm{b}}}\right)=\frac{\mathrm{K}_{\mathrm{w}}}{\left[\mathrm{H}^{+}\right]}+\left(\frac{\alpha_{1} \cdot \mathrm{C}_{\mathrm{a}} \cdot \mathrm{V}_{\mathrm{a}}}{\mathrm{V}_{\mathrm{a}}+\mathrm{V}_{\mathrm{b}}}\right)
$$

que pode ser rearranjada para dar

$$
\left(\left[\mathrm{H}^{+}\right]-\frac{\mathrm{K}_{\mathrm{w}}}{\left[\mathrm{H}^{+}\right]}\right)+\left(\frac{\mathrm{C}_{\mathrm{b}} \cdot\left(\mathrm{V}_{\mathrm{b}}-\alpha_{1} \cdot \mathrm{V}_{\mathrm{eq}}\right)}{\mathrm{V}_{\mathrm{a}}+\mathrm{V}_{\mathrm{b}}}\right)=0
$$

Esta equação é em tudo similar à equação (1.4) com a diferença do aparecimento da fracção molar da forma ionizada $\left(\alpha_{1}\right)$. Devido a esta diferença, a equação (2.3) corresponderá a uma equação de terceiro grau, difícil de resolver em ordem à concentração hidrogeniónica.

Ainda quanto ao balanço de carga, eq.(2.1), a forma ácida pode ser neutra ( $\mathrm{HA}$, como por exemplo $\mathrm{CH}_{3} \mathrm{COOH}$ ) ou possuir carga positiva $\left(\mathrm{HA}^{+}\right.$, como

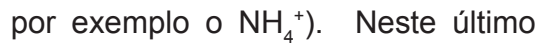
caso o balanço de carga será

$$
\left[\mathrm{H}^{+}\right]+\left[\mathrm{M}^{+}\right]+\left[\mathrm{HA}^{+}\right]=\left[\mathrm{OH}^{-}\right]
$$

onde a concentração da forma ácida é dada por

$$
\begin{aligned}
& {\left[\mathrm{HA}^{+}\right]=\alpha_{0} \cdot\left(\frac{\mathrm{C}_{\mathrm{a}} \cdot \mathrm{V}_{\mathrm{a}}}{\mathrm{V}_{\mathrm{a}}+\mathrm{V}_{\mathrm{b}}}\right)=} \\
& =\left(1-\alpha_{1}\right)\left(\frac{\mathrm{C}_{\mathrm{a}} \cdot \mathrm{V}_{\mathrm{a}}}{\mathrm{V}_{\mathrm{a}}+\mathrm{V}_{\mathrm{b}}}\right)
\end{aligned}
$$

que substituída em (2.4) vai acabar por originar a equação (2.3).

As espécies ácidas com carga negativa (ex: $\mathrm{HA}^{-}$) correspondem de facto a espécies polipróticas.

Estes casos devem ser tratados com titulação de ácidos polipróticos.
CASO 3 - TitulaçÃo de Ácido FORTE (HX) COM UMA BASE FRACA (B)

Neste caso a reacção química a considerar é

$$
\mathrm{H}^{+}(\mathrm{aq})+\mathrm{B}(\mathrm{aq}) \rightarrow \mathrm{HB}^{+}(\mathrm{aq})
$$

Em qualquer instante da titulação o balanço de cargas é dado por

$$
\left[\mathrm{H}^{+}\right]+\left[\mathrm{HB}^{+}\right]=\left[\mathrm{X}^{-}\right]+\left[\mathrm{OH}^{-}\right]
$$

A concentração da espécie protonada $\left(\mathrm{HB}^{+}\right)$é descrita pela eq.(0.9) originando

$$
\left[\mathrm{H}^{+}\right]+\left(\frac{\alpha_{0} \cdot \mathrm{C}_{\mathrm{b}} \cdot \mathrm{V}_{\mathrm{b}}}{\mathrm{V}_{\mathrm{a}}+\mathrm{V}_{\mathrm{b}}}\right)=\frac{\mathrm{K}_{\mathrm{w}}}{\left[\mathrm{H}^{+}\right]}+\left(\frac{\mathrm{C}_{\mathrm{a}} \cdot \mathrm{V}_{\mathrm{a}}}{\mathrm{V}_{\mathrm{a}}+\mathrm{V}_{\mathrm{b}}}\right)
$$

que pode ser rearranjada para dar o modelo desta curva de titulação

$$
\left(\left[\mathrm{H}^{+}\right]-\frac{\mathrm{K}_{\mathrm{w}}}{\left[\mathrm{H}^{+}\right]}\right)+\left(\frac{\mathrm{C}_{\mathrm{b}} \cdot\left(\alpha_{0} \cdot \mathrm{V}_{\mathrm{b}}-\mathrm{V}_{\mathrm{eq}}\right)}{\mathrm{V}_{\mathrm{a}}+\mathrm{V}_{\mathrm{b}}}\right)=0
$$

De igual modo, esta equação não é fácil de ser resolvida por via analítica.

No caso da base fraca possuir carga negativa (MB, como por exemplo $\mathrm{Na}$ $\mathrm{CH}_{3} \mathrm{COO}$ ), o balanço de cargas aplicado à solução do titulado vem

$$
\left[\mathrm{H}^{+}\right]+\left[\mathrm{M}^{+}\right]=\left[\mathrm{OH}^{-}\right]+\left[\mathrm{X}^{-}\right]+\left[\mathrm{B}^{-}\right]
$$

Seguindo a notação escolhida, a concentração da espécie protonada é dada por

$$
\begin{aligned}
& {\left[\mathrm{H}^{+}\right]+\left(\frac{\mathrm{C}_{\mathrm{b}} \cdot \mathrm{V}_{\mathrm{b}}}{\mathrm{V}_{\mathrm{a}}+\mathrm{V}_{\mathrm{b}}}\right)=\frac{\mathrm{K}_{\mathrm{w}}}{\left[\mathrm{H}^{+}\right]}+} \\
& +\left(\frac{\mathrm{C}_{\mathrm{b}} \cdot \mathrm{V}_{\mathrm{eq}}}{\mathrm{V}_{\mathrm{a}}+\mathrm{V}_{\mathrm{b}}}\right)+\left(\frac{\alpha_{1} \cdot \mathrm{C}_{\mathrm{b}} \cdot \mathrm{V}_{\mathrm{b}}}{\mathrm{V}_{\mathrm{a}}+\mathrm{V}_{\mathrm{b}}}\right)
\end{aligned}
$$

que pode ser rearranjada para dar

$$
\left(\left[\mathrm{H}^{+}\right]-\frac{\mathrm{K}_{\mathrm{w}}}{\left[\mathrm{H}^{+}\right]}\right)+\left(1-\alpha_{1}\right) \cdot\left(\frac{\mathrm{C}_{\mathrm{b}} \cdot \mathrm{V}_{\mathrm{b}}}{\mathrm{V}_{\mathrm{a}}+\mathrm{V}_{\mathrm{b}}}\right)-
$$$$
-\left(\frac{\mathrm{C}_{\mathrm{b}} \cdot \mathrm{V}_{\mathrm{eq}}}{\mathrm{V}_{\mathrm{a}}+\mathrm{V}_{\mathrm{b}}}\right)=0
$$

e que é equivalente à anterior equação (3.3).
Caso 4 - Titulação de Ácido fraco (HA) COM UMA BASE FRACA (B)

Este tipo de titulação não se encontra vulgarmente descrito já que tem pouco interesse analítico ${ }^{14}$.

A reacção química a considerar é

$$
\begin{aligned}
& \mathrm{HA}(\mathrm{aq})+\mathrm{B}(\mathrm{aq}) \rightarrow \mathrm{A}^{-}(\mathrm{aq})+ \\
& +\mathrm{HB}^{+}(\mathrm{aq})
\end{aligned}
$$

Em qualquer instante da titulação o balanço de cargas é dado por

$$
\left[\mathrm{H}^{+}\right]+\left[\mathrm{HB}^{+}\right]=\left[\mathrm{OH}^{-}\right]+\left[\mathrm{A}^{-}\right]
$$

Para que se possa distinguir as fracções molares do ácido e da base vão ser acrescentados índices $a$ e $b$ respectivamente. Assim, teremos a equação do balanço de carga sob a forma

$$
\left[\mathrm{H}^{+}\right]+\left(\frac{\alpha_{0 \mathrm{~b}} \cdot \mathrm{C}_{\mathrm{b}} \cdot \mathrm{V}_{\mathrm{b}}}{\mathrm{V}_{\mathrm{a}}+\mathrm{V}_{\mathrm{b}}}\right)=\frac{\mathrm{K}_{\mathrm{w}}}{\left[\mathrm{H}^{+}\right]}+\left(\frac{\alpha_{\mathrm{a}} \cdot \mathrm{C}_{\mathrm{a}} \cdot \mathrm{V}_{\mathrm{a}}}{\mathrm{V}_{\mathrm{a}}+\mathrm{V}_{\mathrm{b}}}\right)
$$

que pode ser rearranjada para dar a equação do modelo que descreve esta curva de titulação

$$
\left(\left[\mathrm{H}^{+}\right]-\frac{\mathrm{K}_{\mathrm{w}}}{\left[\mathrm{H}^{+}\right]}\right)+\left(\frac{\mathrm{C}_{\mathrm{b}} \cdot\left(\alpha_{0 \mathrm{~b}} \cdot \mathrm{V}_{\mathrm{b}}-\alpha_{1 \mathrm{a}} \cdot \mathrm{V}_{\mathrm{eq}}\right)}{\mathrm{V}_{\mathrm{a}}+\mathrm{V}_{\mathrm{b}}}\right)=0
$$

\section{Generalização e Modo de Resolução}

Comparando as equações dos modelos para os quatro casos distintos aqui deduzidos salienta-se a existência de uma grande similaridade entre estes. Verifica-se também que o modelo previsto para o caso 4, eq.(4.3), corresponde a uma situação genérica capaz de satisfazer as situações anteriores, expressas através das equações (1.4), (2.3) e (3.3), quando as fracções molares $\left(\alpha_{0}, \alpha_{1}\right)$ dos electrólitos fortes são unitárias.

Assim sendo pode-se agora definir o modelo genérico de uma curva de titulação através de

$$
\left(\left[\mathrm{H}^{+}\right]-\frac{\mathrm{K}_{\mathrm{w}}}{\left[\mathrm{H}^{+}\right]}\right)+\phi^{*}=0
$$

onde a fracção titulada $\left(\phi^{*}\right)$ assume a forma 


$$
\phi^{*}=\left(\frac{\mathrm{C}_{\mathrm{b}} \cdot\left(\alpha_{0 \mathrm{~b}} \cdot \mathrm{V}_{\mathrm{b}}-\alpha_{1 \mathrm{a}} \cdot \mathrm{V}_{\mathrm{eq}}\right)}{\mathrm{V}_{\mathrm{a}}+\mathrm{V}_{\mathrm{b}}}\right)
$$

No caso 1 , a previsão da curva de titulação é directa e resolvida analiticamente. Nos restantes casos, o algoritmo baseia-se na resolução aproximativa das equações (2.3), (3.3) e (4.3) por iteração: a) inicialmente assume-se um valor de $\mathrm{pH}$ em cada ponto da curva de titulação, b) calcula-se a concentração hidrogeniónica em cada instante da titulação, eq.(0.5), c) estima-se a respectiva fracção titulada, eq.(5.2), d) calcula-se o valor previsto pelo modelo, eq.(5.1) e e) calcula-se o erro do modelo sob a forma de soma de quadrados (SS),

$$
\mathrm{SS}=\sum\left(\mathrm{e}_{\mathrm{i}}\right)^{2}=\sum\left(\left[\mathrm{H}^{+}\right]-\frac{\mathrm{K}_{\mathrm{w}}}{\left[\mathrm{H}^{+}\right]}+\phi^{*}\right)^{2}
$$

As iterações são conduzidas por alteração do valor de $\mathrm{pH}$ no sentido de minimizar o erro do modelo, eq.(5.3).

\section{AplicaÇÃo}

Vejamos agora os quatro casos aplicados, calculados com base numa foIha de cálculo.

Para os menos familiarizados com este tipo de software recomenda-se a consulta de livros da especialidade $[4,5]$.

Neste trabalho vamos ilustrar alguns dos casos obtidos no estudo das quatro situações em estudo, titulação de a) ácido forte $(25,00 \mathrm{~mL} \mathrm{HCl} 0,100 \mathrm{M})$ com uma base forte $(\mathrm{NaOH} 0,100 \mathrm{M})$, b) ácido fraco $\left(25,00 \mathrm{~mL} \mathrm{CH} \mathrm{CH}_{3} \mathrm{COOH}\right.$ $\left.0,100 \mathrm{M}, \mathrm{pK}_{\mathrm{a}}=4,75\right)$ com uma base forte $(\mathrm{NaOH} \mathrm{0,100} \mathrm{M),} \mathrm{c)} \mathrm{ácido} \mathrm{forte}$ (25,00 mL HCl 0,100 M) com uma base fraca $\left(\mathrm{NH}_{3} 0,100 \mathrm{M}, \mathrm{pK}_{\mathrm{a}}=9,26\right)$ e d) ácido fraco $\left(25,00 \mathrm{~mL} \mathrm{CH}_{3} \mathrm{COOH}\right.$ $\left.0,100 \mathrm{M}, \mathrm{pK}_{\mathrm{a}}=4,75\right)$ com uma base fraca $\left(\mathrm{NH}_{3} 0,100 \mathrm{M}, \mathrm{pK}_{\mathrm{a}}=9,26\right)$.

Utilizar uma folha de cálculo (Excel) permite, entre outras vantagens, utilizar um poderosa ferramenta de optimização (suplemento SOLVER) e obter uma aceitável representação gráfica que é automaticamente actualizada após cada cálculo. Esta "macro" do Excel recorre ao algoritmo GRG2 ${ }^{15}$ que é adequado para a optimização de problemas envolvendo funções não lineares $[5,6]$.

\section{Procedimento}

De seguida descrevemos as condições de utilização da folha de cálculo e mostraremos alguns resultados conseguidos. Para que o leitor possa relacionar a disposição dos valores na respectiva folha de cálculo, na figura 1 encontra-se uma imagem parcial de um exemplo de folha de cálculo onde foram realizados os cálculos da estimativa da curva de titulação (caso 2). sempre 25,00 mL. Para se obter uma boa curva de titulação deve-se ultrapassar o ponto de equivalência em pelo menos $50 \%$ do valor de equivalência o que corresponde ao volume final de $37,50 \mathrm{~mL}$. No sentido de representar melhor a curva no ramo alcalino escolheu-se o valor final de $40,00 \mathrm{~mL}$. O incremento para volume de titulação deve ser escolhido em conformidade com a necessidade de cada utilizador. Um incremento constante não é a escolha mais sensata se este for demasiado grande. No caso testado foi escolhido um incremento constante de $0,50 \mathrm{~mL}$

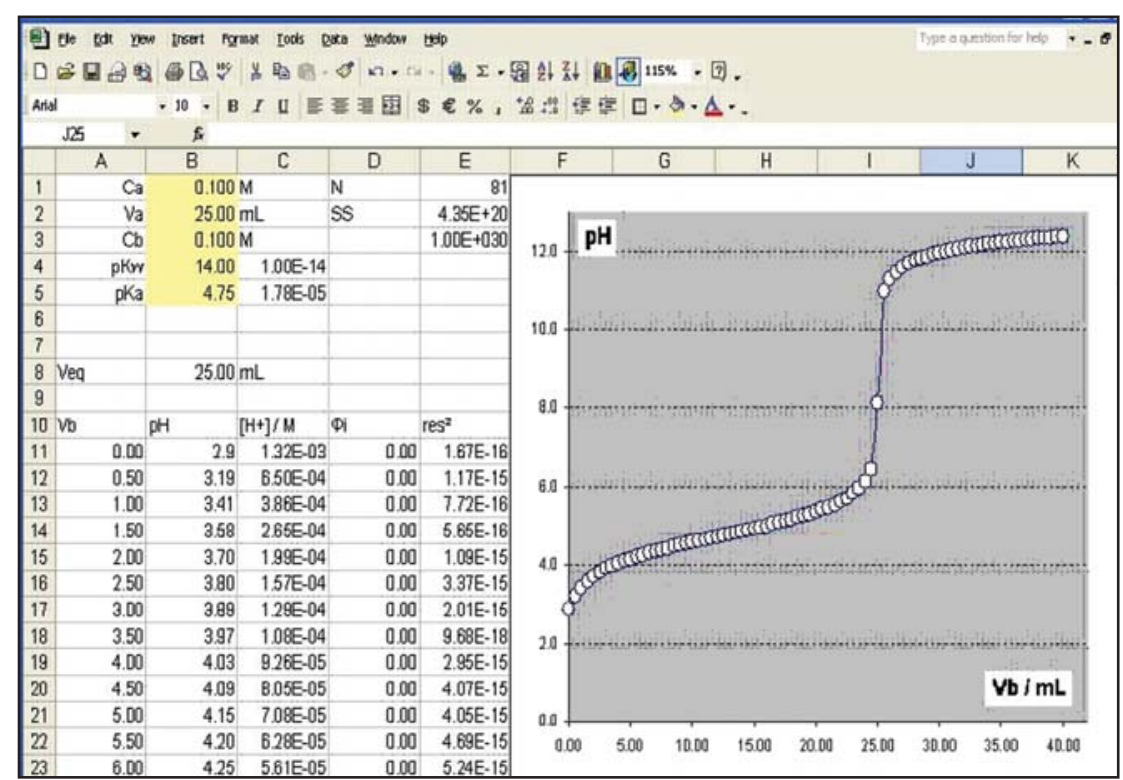

Figura 1 Imagem parcial da folha de cálculo utilizada para o caso 2: destaque, com fundo amarelado, do local onde devem ser inseridos os parâmetros experimentais referentes à curva da titulação

Para facilitar, cada titulação deve ser processada numa folha de cálculo independente. $\mathrm{O}$ procedimento resume-se a:

1. Inserir os valores da concentração do ácido $\left(\mathrm{C}_{\mathrm{a}}\right.$, célula $\left.\mathrm{B} 1\right), \mathrm{O}$ volume pipetado $\left(V_{a}\right.$, cél. $\left.B 2\right)$, e concentração do titulante $\left(\mathrm{C}_{\mathrm{b}}\right.$, cél. B3) - o valor do volume de equivalência $\left(V_{\text {eq }}\right.$, cél. $B 8=B 1{ }^{*} B 2 /$ B3) é automaticamente calculado com base nestes dados;

2. Colocar o valor das constantes de equilíbrio: o produto iónico da água ( $\mathrm{pKw}$, cél. B4, convertido na respectiva constante na célula C6 através de "=10^(-B4)"); e a constante de acidez ( $\mathrm{pK}_{\mathrm{a}}$, cél. B5);

3. Deve-se definir inicialmente o escala de volume a utilizar - nestes casos o volume equivalente é
( $2 \%$ do volume equivalente). Os respectivos valores estão localizados na folha de cálculo na coluna A (céls. A11:A91);

4. A coluna B (céls. B11:B91) destinase às estimativas de $\mathrm{pH}$. Estas primeiras estimativas vão afectar a rapidez de convergência mas, se não se quiser ter trabalho extra, pode-se inclusivamente assumir em todos os pontos o valor 7,00 .

5. A coluna C (céls. C11:C91) destina-se ao valor da concentração hidrogeniónica respectiva. A fórmula inscrita em C11 é "=10^(-B11)" e é estendida a toda a coluna até ao endereço C91;

6. A coluna D (céls. D11:D91) destinase à estimativa da fracção titulada $\left(\phi^{*}\right)$, eq.(5.2). A fórmula introduzida no caso 2 em $D 11$ é " $=\$ B \$ 3^{*}(A 11-$ $\$ B \$ 8 *(\$ C \$ 5 /(C 11+\$ C \$ 5))) /$ 
$(\$ B \$ 2+A 11) " 16 ;$

7. A coluna $E$ (céls. E11:E91) destinase à estimativa do erro do modelo em cada ponto (e $e_{\mathrm{i}}$, eq.(5.3). A fórmula inserida em E11 é " $=((C 11-$ $\$ C \$ 4 / C 11)+D 11)^{\wedge} 2$ e e esta é estendida à restante coluna até ao endereço E91;

8. A soma de quadrados (SS), eq.(5.3), é calculada na própria folha de cálculo (cél. E2). Um pequeno comentário deve aqui ser incluído. A soma de quadrados corresponde a uma "hipersuperfície"17 onde a ferramenta de optimização procura orientar a sua tarefa no sentido de localizar o mínimo desta superfície. Dado ainda que o Excel trabalha com inteiros de dupla precisão (com 15 algarismos significativos), espera-se que o erro de truncatura numérica se situe próximo de $10^{-15}$. A soma de quadrados deve então atingir um mínimo absoluto da ordem de $10^{-30}$ o que conduz a uma superfície excepcionalmente plana para ser localizado eficientemente o mínimo. Para tornar a busca mais eficiente pode-se multiplicar a soma de quadrados por uma constante (ex: $10^{30}$, cél. E3) e contornar esta insensibilidade;

9. Inicia-se a optimização activando o SOLVER, procurando minimizar o erro do modelo (cél. E2, que contém a fórmula "=SOMA(E11:E91)*E3") por alteração do valor do pH (céls. B11:B91). As opções escolhidas na optimização são cruciais para o tempo de cálculo e sucesso da convergência.

$\mathrm{Na}$ figura 2 encontra-se um detalhe sobre a caixa de diálogo do SOLVER: em a) indica-se os objectivos para optimização e em b) definem-se as opções de convergência (o acesso a esta caixa de diálogo é conseguido através do botão "options").

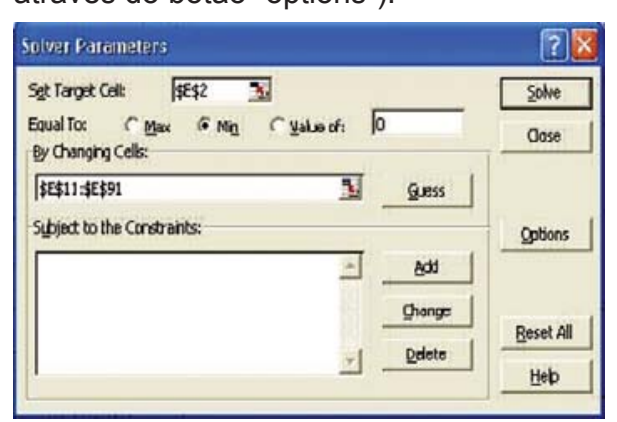

a) condições para optimização
Regra geral verifica-se que basta apenas um ciclo completo de iterações para atingir o mínimo global que corresponde às estimativas mais correctas. Contudo, recomenda-se [6] que, nestes casos, após ter obtido o valor mínimo para a função de erro, se preserve o resultado final (curva de titulação e respectivo erro, SS) e se reinicie o processo com outras estimativas iniciais para verificar se se consegue obter outro mínimo da função de erro que possua um valor inferior ao anterior - nesse caso considera-se que esta última estimativa está mais correcta.

Neste trabalho verificou-se que, se as condições iniciais escolhidas para a optimização forem razoáveis e a solução final for de novo testada até não se ser capaz de optimizar mais, o valor final do erro obtido por convergência pouco difere entre procedimentos, indicando que 0 SOLVER é uma ferramenta muito poderosa na busca robusta de mínimos da função de erro. Contudo, a sua velocidade de cálculo depende essencialmente de cinco factores cruciais:

1. Do tipo de computador utilizado (características do processador de cálculo e quantidade de memória RAM disponível) e da versão do software utilizado - maiores capacidades de processamento, como por exemplo, maiores frequências de processamento e/ ou processadores em paralelo, reduzem drasticamente o tempo de cálculo. Neste trabalho tivemos a oportunidade de testar as versões MS Excel 2002 em processadores relativamente antigos (AMD $350 \mathrm{MHz}$

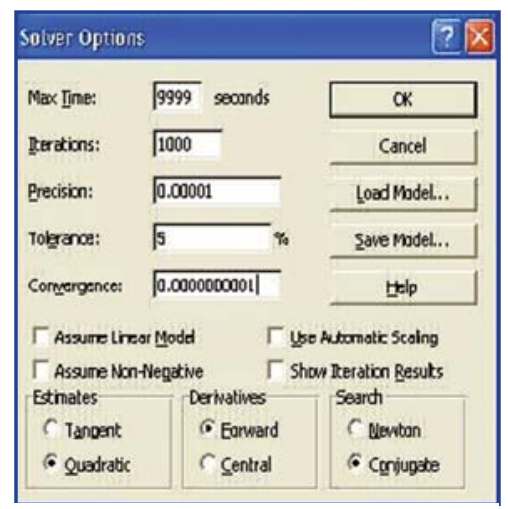

b) opções de optimização

Figura 2 Imagem ilustrativa das fases de activação da ferramenta de optimização SOLVER: a) detalhe com indicação das condições de optimização e b) opções de optimização para a convergência com 128 MB de RAM) e (AMD 800 $\mathrm{MHz}$ com $128 \mathrm{MB}$ de RAM) a correr sobre o Windows XP Professional e o MS Excel 2007 num (AMD Turion X2 2,0 GHz (dual core) com 3,0 Gb de RAM) a correr sobre o sistema operativo Windows Vista. Destaca-se uma melhoria significativa no tempo de cálculo com processadores mais potentes. Em termos ilustrativos, os primeiros 100 ciclos de iteração do caso 2 levaram, respectivamente, cerca de 51,32 e 9 segundos a serem executados em cada sistema testado;

2. Do número de pontos utilizados para o cálculo da curva de titulação - curvas mais detalhadas requerem uma maior densidade de pontos que solicitam mais cálculo ao processador, atrasando a convergência do algoritmo;

3. Do número de equilíbrios envolvidos no sistema - maior número de equilíbrios dificulta o cálculo e requer mais tempo de processamento. Por exemplo o Caso 2 requer cerca de $1 / 3$ das iterações do Caso 4;

4. Das estimativas iniciais indicadas - o valor de $\mathrm{pH}$ da aproximação inicial quanto mais afastado estiver do valor final mais iterações requer para a convergência;

5. Dos parâmetros de optimização escolhidos para o SOLVER verificou-se que estes parâmetros são muito importantes na velocidade de convergência. Efectuando alguns ensaios verificou-se que é preferível tentar fazer o cálculo iterativo de uma só vez, sem qualquer interrupção, pois desta forma o algoritmo utilizado consegue evitar melhor mínimos locais $^{18}$. Assim, é necessário providenciar no sentido de não restringir o cálculo quer por limite de tempo quer por número insuficiente de iterações.

Regra geral os seguintes parâmetros: tempo máximo $=9999 \mathrm{~s}$, iterações $=$ 1000 , precisão $=0,00001$, tolerância $=$ 5 , convergência $=0,000000001$, com as opções estimativas quadráticas, derivadas posteriores, e pesquisa conjugada, permitem um cálculo eficiente e garantem uma boa busca da solução do problema. Estas opções 
são definidas após seleccionar o botão de "Options" do SOLVER, ver figura 2 .

É ainda recomendado que, após a convergência, se reinicie o SOLVER no sentido de verificar se este consegue ou não melhorar as estimativas obtidas.

Na figura 3 encontra-se representada a evolução da forma da curva de titulação (caso 2) em função do número de iterações realizadas.

Da figura 3 verifica-se que, com as condições inicialmente impostas, com um número relativamente modesto de iterações (200) a curva final da titulação já está significativamente bem definida sendo, contudo, necessárias mais iterações para atingir o aspecto final, figura 4, ao fim de cerca de 800 iterações.

A título de exemplo apresenta-se ainda na figura 5 o traçado da curva de titulação referente à titulação de um ácido

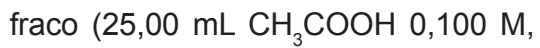
$\left.\mathrm{pK}_{\mathrm{a}}=4,75\right)$ com uma base fraca $\left(\mathrm{NH}_{3}\right.$ $\left.0,100 \mathrm{M}, \mathrm{pK}_{\mathrm{a}}=9,26\right)$, caso 4 .

\section{Notas}

1 Estas curvas de titulação exprimem, regra geral, o pH de uma solução em função do volume adicionado de titulante.

2 A carbonatação de soluções aquosas deve-se à dissolução do dióxido de carbono $\left(\mathrm{CO}_{2}\right)$ atmosférico na mistura. $\mathrm{O}$ ácido carbónico $\left(\mathrm{H}_{2} \mathrm{CO}_{3}\right)$ formado ioniza-se para dar bicarbonato $\left(\mathrm{HCO}_{3}{ }^{-}\right)$ e como consequência acidifica a solução. Enquanto uma solução possuir valores de $\mathrm{pH}$ baixo $(<4)$ a carbonatação é contrariada já que a ionização do ácido carbónico é minimizada, contudo, em soluções com valores de $\mathrm{pH}$ superiores a 5 a dissolução deste gás é promovida pela ionização do respectivo ácido.

3 O titulante é a solução que se encontra dentro da bureta e que vai ser controlada através do volume vertido.

4 O titulado é a solução que se encontra dentro do recipiente que recebe o titulante despejado pela bureta.

5 No caso de titulações mais demoradas é necessário possuir um sistema com purga contínua de gás inerte (ex: azoto ultrapuro).

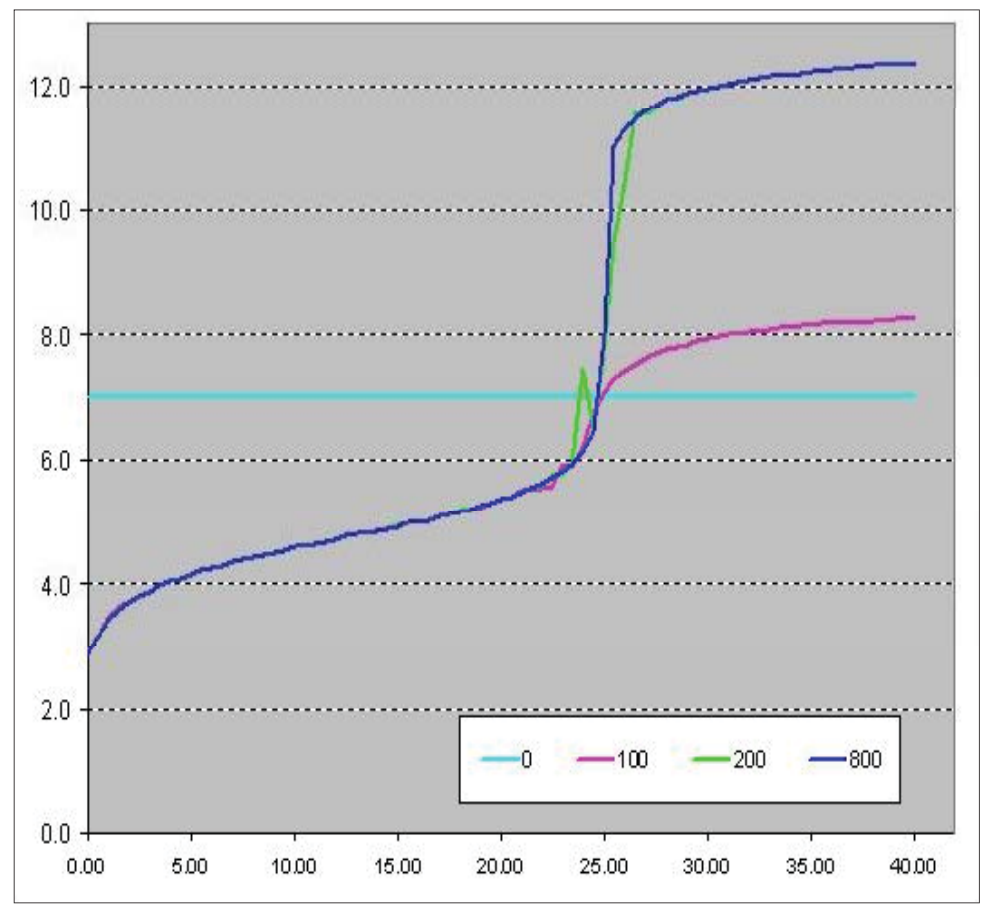

Figura 3 Evolução das estimativas da curva de titulação (caso 2) de ácido fraco $(25,00 \mathrm{~mL}$ de $\left.\mathrm{CH}_{3} \mathrm{COOH} \mathrm{0,100M,} \mathrm{pK}=4,75\right) \mathrm{com}$ base forte $(\mathrm{NaOH} 0,100 \mathrm{M})$ em função do número

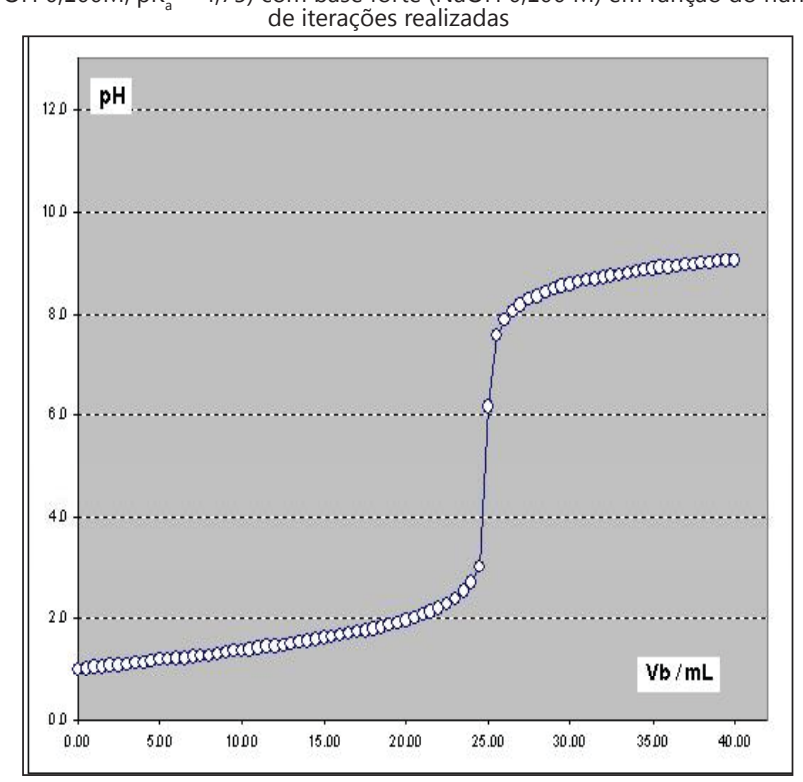

Figura 4 Aspecto final da curva de titulação de ácido fraco $\left(25,00 \mathrm{~mL}\right.$ de $\mathrm{CH}_{3} \mathrm{COOH} 0,100 \mathrm{M}$, $\mathrm{pKa}=4,75)$ com base forte $(\mathrm{NaOH} 0,100 \mathrm{M})$

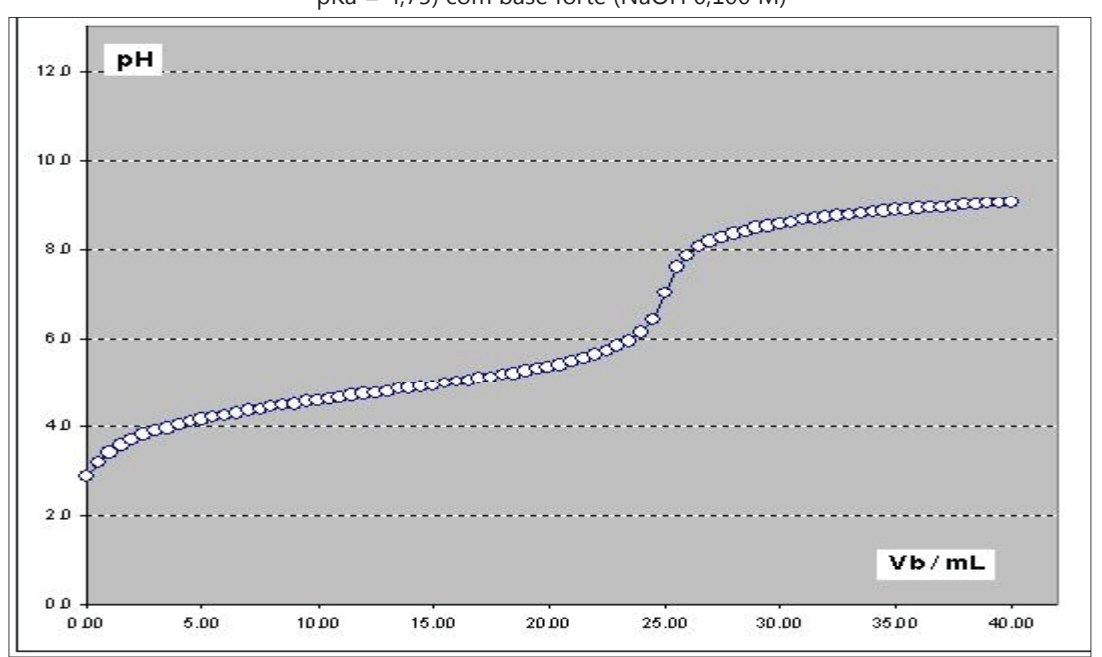

Figura 5 Curva de titulação calculada para o doseamento do ácido fraco $(25,00 \mathrm{~mL}$ de $\mathrm{CH}_{3} \mathrm{COOH} 0,100 \mathrm{M}$, pKa $\left.=4,75\right) \mathrm{com}$ base fraca $\left(\mathrm{NH}_{3} 0,100 \mathrm{M}, \mathrm{pKa}=9,26\right)$ 
6 Uma agitação adequada promove a rápida homogenização sem causar perdas de solução nem promover demasiada contaminação da solução.

7 Espécies monopróticas são ácidos ou bases que apenas podem ceder/captar um protão, respectivamente.

8 A zona tamponizante de uma curva de titulação revela-se por ter um baixo valor de declive $(|\mathrm{dpH} / \mathrm{dV}|$, em valor absoluto) indicando a resistência da solução a variações de $\mathrm{pH}$ promovidas pelo titulante adicionado. Esta propriedade deve-se à existência de um equilíbrio ácido-base na solução onde estão presentes em concentração significativa as respectivas formas ácido e base conjugada.

9 Volume de equivalência é o volume de titulante que corresponde estequiometricamente ao titulado utilizado.

10 O ponto de viragem é a região da curva de titulação onde o declive atinge maior valor absoluto e corresponde à proporção estequiométrica entre os reagentes titulados.

11 O que implica considerar constantes a temperatura, a pressão e a força iónica.

12 As unidades utilizadas são irrelevantes - apenas se exige que estas sejam todas coerentes. No caso de dúvida, a molaridade (M) deve ser usada para as concentrações das espécies e mililitros $(\mathrm{mL})$ para os volumes.

13 Para melhorar a resolução da curva de titulação aconselha-se aumentar a densidade de pontos na região próxima do ponto de equivalência, onde o $\mathrm{pH}$ varia mais bruscamente.

14 Esta titulação apresenta uma pequena variação de $\mathrm{pH}$ no ponto de viragem o que conduzirá a erros de quantificação significativos.

15 A sigla GRG2 significa Generalized Reduced Gradient. Trata-se de um método de pesquisa de soluções com base em gradientes da função de mérito.

${ }^{16}$ Nas folhas de cálculo utiliza-se o símbolo "\$" para fixar a referência em relação à coluna (se estiver antes da respectiva letra) e/ou em relação ao número da linha (se estiver à esquerda desta), por exemplo, $\$ B \$ 3$ é a referência inalterável sobre a célula B3, onde se encontra, nestes casos, o valor da concentração da base, $C_{b}$.

17 A soma de quadrados no caso de um modelo com apenas duas variáveis ajustadas assemelha-se a uma superfície côncava cujo mínimo corresponde à melhor estimativa paramétrica para o modelo. No caso de o número de parâmetros de ajuste ser superior a dois, esta superfície de erro não pode ser representada graficamente numa perspectiva tridimensional, chamando-se por esta razão de hipersuperfície.

18 Num processo iterativo de optimização baseado na pesquisa guiada por gradiente da função de erro, é frequente serem localizadas situações onde alguns dos gradientes se anulam indicando a presença de valores mínimos locais que não correspondem à situação final, onde todos os parâmetros de ajuste estão correctamente ajustados.

Estas ferramentas de cálculo podem ser obtidas mediante solicitação dos interessados por via e-mail para jcpereira@qui.uc.pt sob o assunto "Previsão de curvas de titulação: folha de cálculo PrevCTPMono.xls".

\section{REFERÊNCIAS}

[1] R. Chang, Chemistry, $8^{a}$ Ed., McGraw-Hill, New York, 2004.

[2] P. Atkins and L. Jones, Chemical Principles: the quest for insight, $3^{\text {th }}$ Ed., Freeman, New York, 2005.

[3] J.N. Butler, Ionic equilibrium: a mathematical approach, Addison-Wesley, London, 1964.

[4] R. Maran, Maran Illustrated Excel 2003, Maran Illustrated Series, Premier Pr, London, 2005.

[5] E.J. Billo, Excel for Scientists and Engineers Numerical Methods, Wiley, 2007.

[6] D. Fylstra, L. Lasdon, J. Watson, A. Waren, Interfaces 28 (1998) 29-55.

\section{Actualidade Científica}

\section{Depósitos de CARBonato ENCONTRA- DOS EM MARTE}

Após uma longa pesquisa de décadas foi anunciada a descoberta de depósitos substanciais de rochas carbonatadas em Marte, mais concretamente, de carbonato de magnésio, numa área inferior a dez quilómetros quadrados.

Estas rochas não estão associadas ao leito de oceanos já desaparecidos (como se esperou em tempos) mas podem conter resíduos de matéria orgânica associada à história do planeta. Os detalhes da descoberta são descritos na revista Science 322 (2008) 1828-1832 por B. L. Ehlmann e colaboradores. Para explicar a atmosfera fina de Marte tem sido prevista a existência de carbonatos na crosta de Marte. Supõe-se que, na presença de água e de rochas contendo silicatos, o dióxido de carbono da atmosfera seria convertido em carbonatos.

Apesar de haver algumas experiências que mostravam evidência para a sua existência, não havia de facto observação de rochas carbonatadas na superfície. As observações da "Mars Reconnaissance Orbiter" (MRO) per- mitiram confirmar, por análise de espectros de Infra-Vermelho, a presença de uma camada rochosa contendo carbonato de magnésio. A maioria dos minerais criados à superfície de Marte por interacção com água parecem resultar de meios fortemente acídicos. A descoberta de rochas carbonatadas indica a existência de condições mais alcalinas, possivelmente mais propícias à vida.

(Adaptado de Nature News de 19 de Dezembro de 2008) 
Most accessed articles 9/2006 - 8/2007

A General and Facile One-Step Synthesis of Imido-Titanium(IV) Complexes: Application to the Synthesis of Compounds Containing Functionalized or Chiral Imido Ligands and Bimetallic Diimido Architectures C. Lorber, R. Choukroun, L. Vendier Eur. J. Inorg. Chem. 2006, 4503-4518 Classical and Non-Classical RutheniumBased Anticancer Drugs: Towards Targeted Chemotherapy

W. H. Ang, P. J. Dyson

Eur. J. Inorg. Chem. 2006, 4003-4018

Nanoscopic Metal Particles - Synthetic Methods and Potential Applications

H. Bönnemann, R. M. Richards

Eur. J. Inorg. Chem. 2001, 2455-2480

Nanoparticulated Gold: Syntheses,

Structures, Electronics, and Reactivities

G. Schmid, B. Corain

Eur. J. Inorg. Chem. 2003, 3081-3098

Osmium- and Ruthenium-Based Phosphorescent Materials: Design, Photophysics, and Utilization in OLED Fabrication

P. - T. Chou, Y. Chi

Eur. J. Inorg. Chem. 2006, 3319-3332

Full-text downloads have more than doubled since 2003. EurJIC is among the top ten most-cited primary journals in inorganic chemistry.

For more information please visit: www.eurjic.org

Subscribe now! Please send an e-mail to:

cs-journals@wiley.com

(North and South America)

service@wiley-vch.de

(Germany/Austria/Switzerland)

cs-journals@wiley.co.uk

(all other areas)

\section{(3) WILEY}

InterScience

WILEY-VCH

\section{Made in Europe for the World}

\section{EurJIC Facts}

- Short Communications median time from submission to web publication 10 weeks

- Author submissions have more than doubled since 2001

- Steady increase in impact since the amalgamation of ten national society journals

- Table of Contents redesigned in 2007 to assist browsing

- Templates available for submission of articles

- Increased frequency in 2007: 36 issues

- Owned and supported by 13 national chemical societies in Europe (Editorial Union of Chemical Societies)

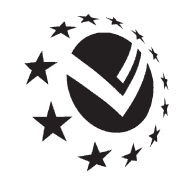

EUChemSoc

\section{EurJIC offers:}

- RSS feeds

- Citation tracking

- Table of Contents Alerts

- Backfiles of Chemische Berichte (1868-1997) as part of the Chemical Societies Backfiles Collection

- Attractive personal subscription rates for society members

\section{International interest in EurJIC as reflected by the percentage of downloads from each country}

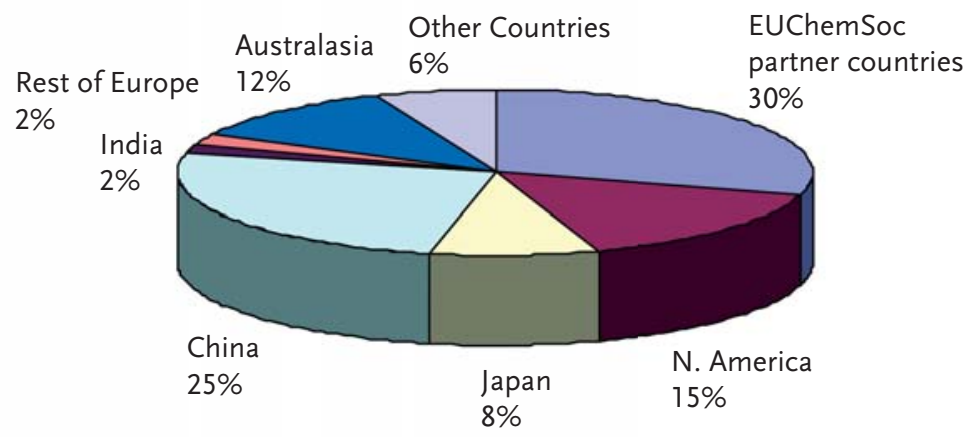

\section{Impact Factors announced in the year given}

1997

2.15

1999

2.221

2001

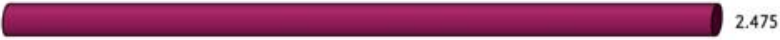

2003

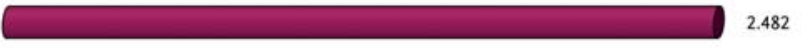

2005

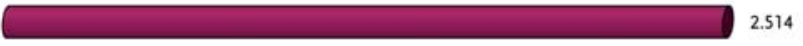

2007 


\section{(अ)WILEY}

Chemistry \& Sustainability, Energy \& Materials

\section{To view the latest articles below, simply visit www.chemsuschem.org}

\section{Communications:}

$\varepsilon$-Caprolactamium Hydrogen Sulfate: An Ionic Liquid Used for Decades in the Large-Scale Production of $\varepsilon$-Caprolactam I. T. Horváth et al.

Highly Active Catalysts for the Telomerization of Crude Glycerol with 1,3-Butadiene

B. M. Weckhuysen et al.

Synthesis of Dimethyl Carbonate from Carbon Dioxide Catalyzed by Titanium Alkoxides with Polyether-type Ligands T. Sakakura et al.

A Versatile Iridium Catalyst for Aldehyde Reduction in Water

J. Xiao et al.

\section{Full Papers:}

Surfactants from Biomass: A Two-Step Cascade Reaction for the Synthesis of Sorbitol Fatty Acid Esters Using Solid Acid Catalysts A. Corma et al.

Regiodivergent Baeyer-Villiger Oxidation of Fused Ketones by Recombinant Whole-Cell Biocatalysts

M. D. Mihovilovic et al.

A Highly Active Aqueous Olefin Metathesis Catalyst Bearing a Quaternary Ammonium Group K. Grela et al.

On the Energy Efficiency of Microwave-Assisted Organic Reactions

C. O. Kappe et al.

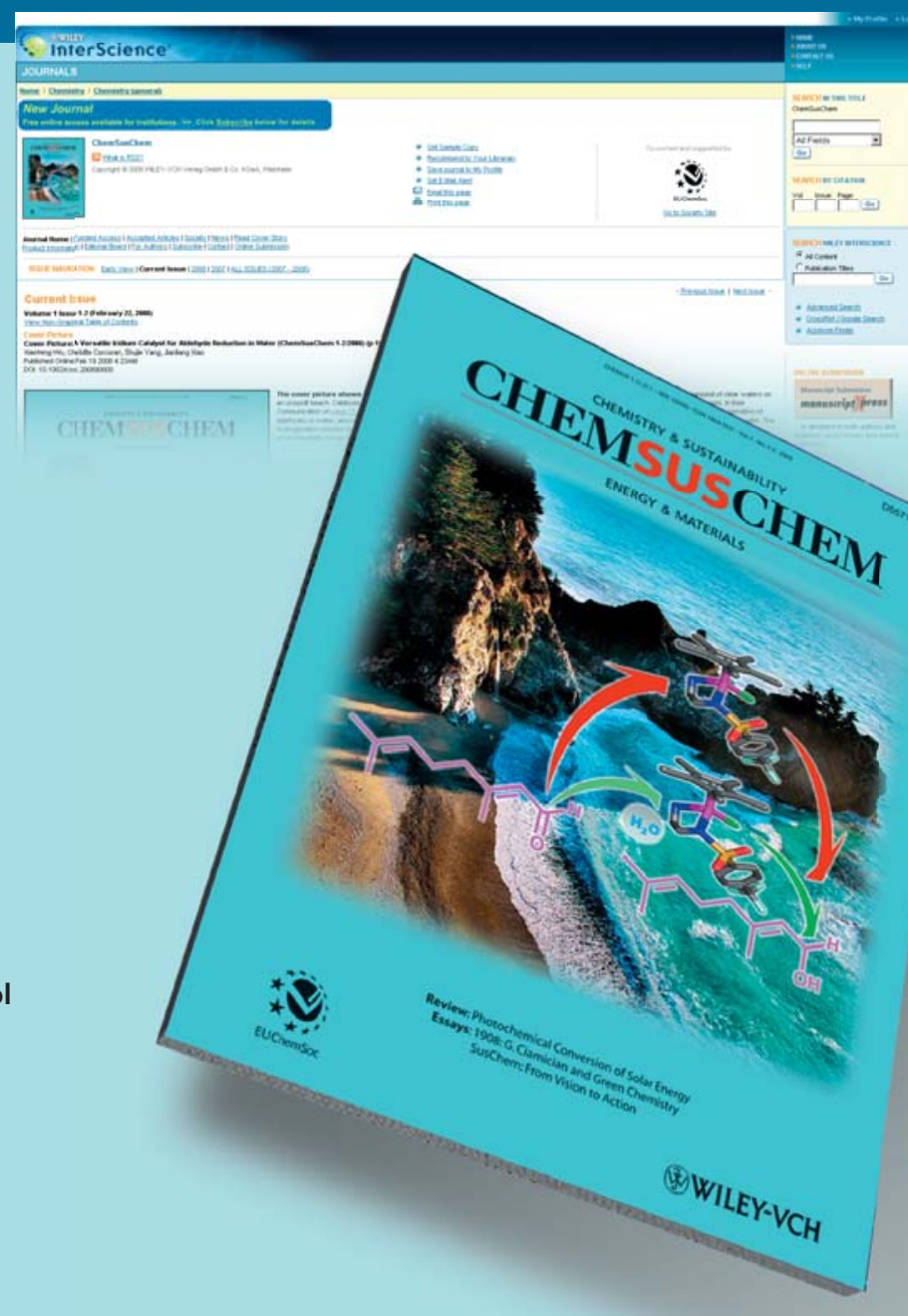

Review:

Photochemical Conversion of Solar Energy V. Balzani et al.

Founding Societies:

Società Chimica Italiana (SCI)

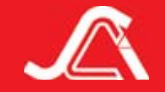

Gesellschaft Deutscher

Chemiker (GDCh)
ChemSusChem is co-owned by Editorial Union of Chemical Societies

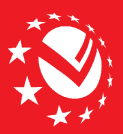
EUChemSoc and supported by Asian Chemical Editorial Society

\section{Subscribe now!}

Institutions can opt to receive Complimentary Online Access to ChemSusChem throughout 2008.

Register here

www.interscience.wiley.com/newjournals

cs-journals@wiley.com (North and South America) service@wiley-vch.de (Germany/Austria/Switzerland) cs-journals@wiley.co.uk (All other areas) 